\title{
Function and clinical relevance of RHAMM isoforms in pancreatic tumor progression
}

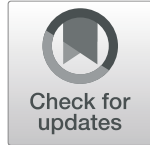

Soyoung Choi ${ }^{1}$, Dunrui Wang ${ }^{2}$, Xiang Chen ${ }^{1}$, Laura H. Tang ${ }^{3}$, Akanksha Verma ${ }^{4}$, Zhengming Chen ${ }^{5}$, Bu Jung Kim', Leigh Selesner ${ }^{1}$, Kenneth Robzyk ${ }^{3}$, George Zhang ${ }^{1}$, Sharon Pang ${ }^{1}$, Teng Han ${ }^{6}$, Chang S. Chan ${ }^{7}$, Thomas J. Fahey III $^{8}$, Olivier Elemento ${ }^{4}$ and Yi-Chieh Nancy Du ${ }^{1,6^{*}}$ (D)

\begin{abstract}
The receptor for hyaluronic acid-mediated motility (RHAMM) is upregulated in various cancers. We previously screened genes upregulated in human hepatocellular carcinomas for their metastatic function in a mouse model of pancreatic neuroendocrine tumor (PNET) and identified that human $R H A M M^{B}$ promoted liver metastasis. It was unknown whether RHAMM ${ }^{B}$ is upregulated in pancreatic cancer or contributes to its progression. In this study, we found that RHAMM protein was frequently upregulated in human PNETs. We investigated alternative splicing isoforms, RHAMM ${ }^{A}$ and $R H A M M^{B}$, by RNA-Seq analysis of primary PNETs and liver metastases. RHAMM ${ }^{B}$, but not RHAMM ${ }^{A}$, was significantly upregulated in liver metastases. RHAMM ${ }^{B}$ was crucial for in vivo metastatic capacity of mouse and human PNETs. RHAMM ${ }^{\mathrm{A}}$, carrying an extra 15-amino acid-stretch, did not promote metastasis in spontaneous and experimental metastasis mouse models. Moreover, RHAMM ${ }^{B}$ was substantially higher than RHAMM ${ }^{A}$ in pancreatic ductal adenocarcinoma (PDAC). RHAMM ${ }^{B}$, but not $R H A M M^{A}$, correlated with both higher EGFR expression and poorer survival of PDAC patients. Knockdown of EGFR abolished RHAMM ${ }^{B}$-driven PNET metastasis. Altogether, our findings suggest a clinically relevant function of $R H A M M^{B}$, but not $R H A M M^{A}$, in promoting PNET metastasis in part through EGFR signaling. RHAMM ${ }^{B}$ can thus serve as a prognostic factor for pancreatic cancer.
\end{abstract}

Keywords: RHAMM, Isoforms, Pancreatic cancer, PNETs, PDAC, Metastasis

\section{Main text}

Metastasis accounts for $90 \%$ of cancer deaths. We developed a mouse model of well-defined multistage tumorigenesis: RIP-Tag; RIP-tva to identify metastatic factors [1]. We identified that the receptor for hyaluronic acid (HA)-mediated motility, isoform B $\left(\right.$ RHAMM $\left.^{\mathrm{B}}\right)$, significantly promotes liver metastasis of pancreatic neuroendocrine tumors (PNET) in RIP-Tag; RIP-tva mouse models [2]. Expression of RHAMM is restricted in normal adult tissues, but is upregulated in cancers [3, 4]. Increased production of glycosaminoglycan, HA, is correlated with increased migration and invasion in aggressive cancers [5]. CD44 and RHAMM are two major

\footnotetext{
* Correspondence: nad2012@med.cornell.edu

'Department of Pathology and Laboratory Medicine, Weill Cornell Medicine, Box 69, New York, NY 10065, USA

${ }^{6}$ Weill Cornell Graduate School of Medical Sciences, Cornell University, New York, NY 10065, USA

Full list of author information is available at the end of the article
}

HA receptors. The roles of CD44 isoforms in cancer have been studied extensively, but the functions of RHAMM isoforms in tumorigenesis are less clear. RHAMM encodes 18 exons and alternative splicing generates different isoforms. $R H A M M^{A}$ includes all 18 exons and $R H A M M^{B}$ lacks exon 4 (Fig. 1a). Here we aimed to determine the clinical relevance of $R H A M M^{A}$ and $R H A M M^{B}$ isoforms and their functions in pancreatic cancer.

\section{RHAMM $^{B}$, but not RHAMM ${ }^{A}$, is upregulated in human PNET liver metastases}

To investigate RHAMM expression in human PNETs, a tissue microarray consisting of 83 PNETs was immunostained for RHAMM. RHAMM was not detectable in the normal pancreas, while 54 of 83 (65\%) PNETs exhibited cytoplasmic staining using an antibody that recognizes common region in RHAMM isoforms (Fig. 1b). Because isoform-specific RHAMM antibodies were not available, we investigated the mRNA levels of $R H A M M^{A}$ 
a exon 4 coding region (15 amino acids)

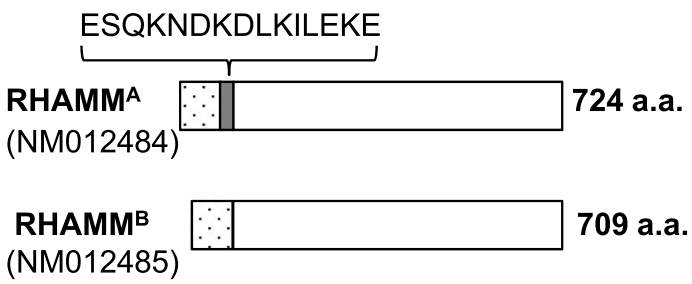

b

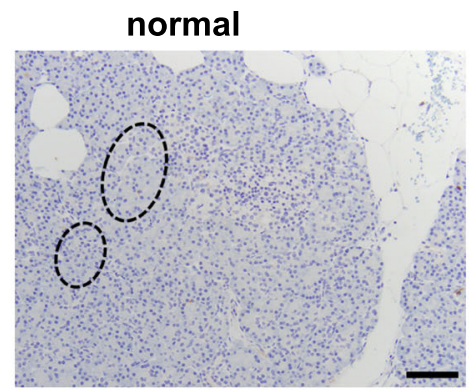

RHAMM (-) tumor

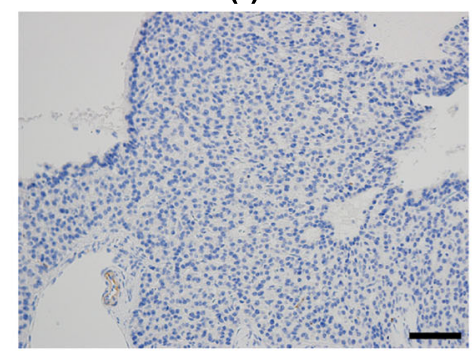

d
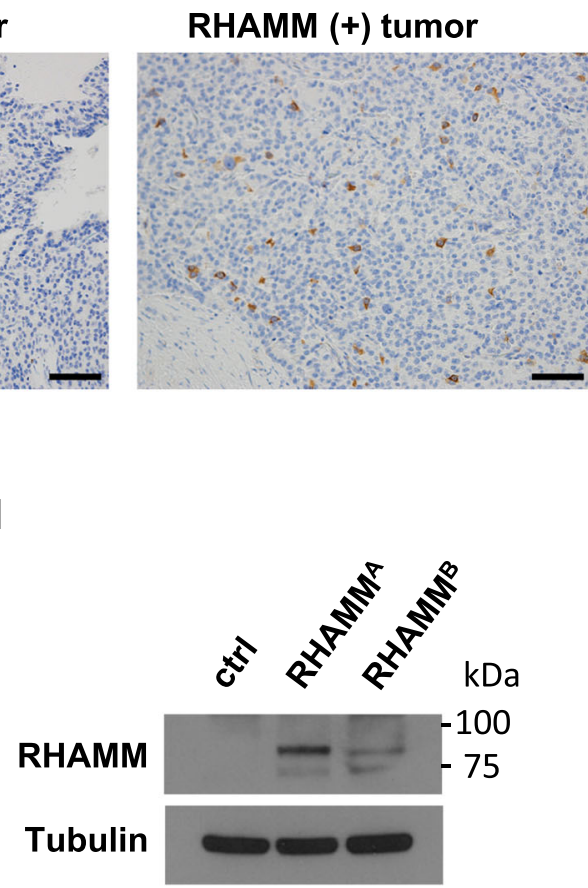

e
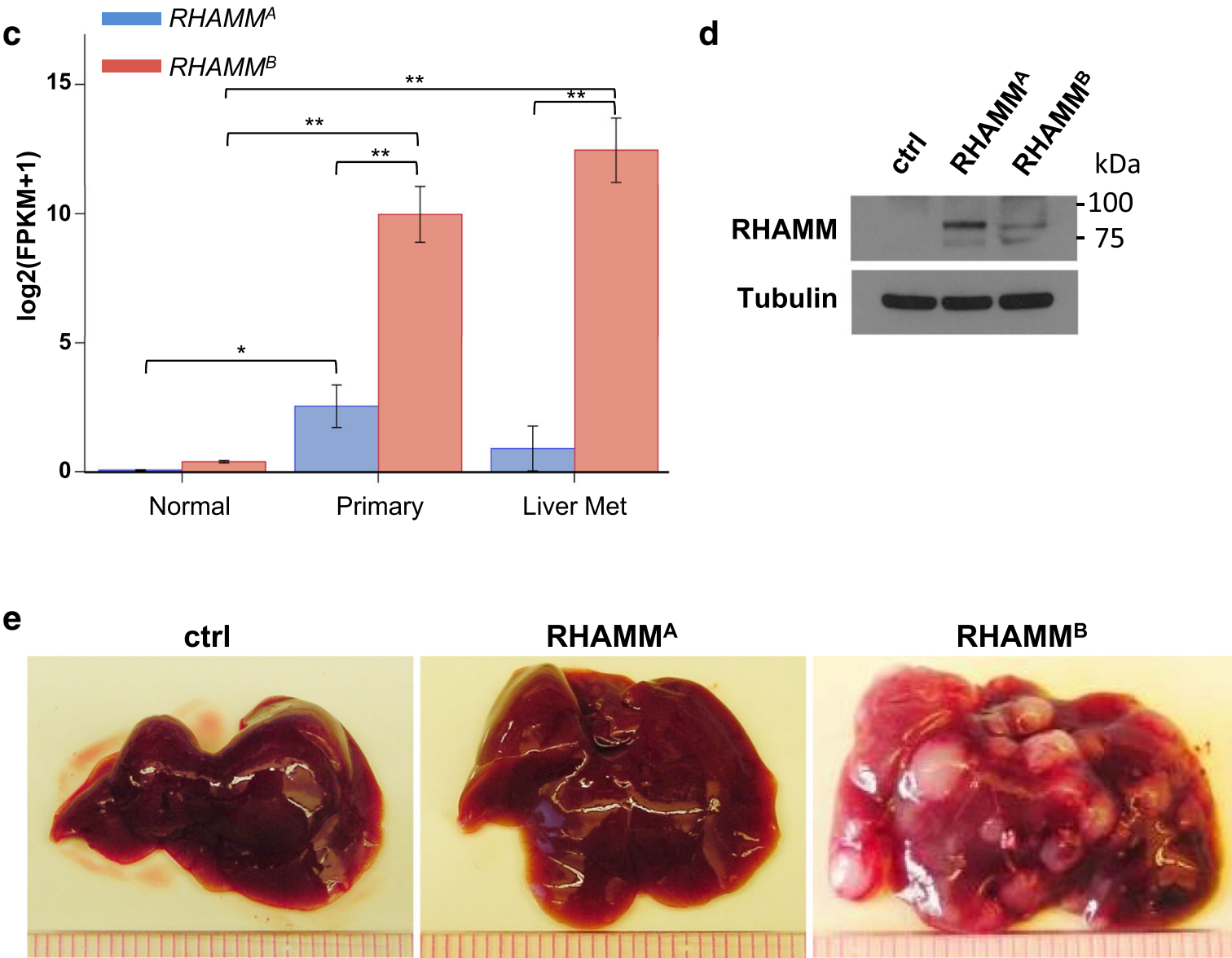

Fig. 1 (See legend on next page.) 
(See figure on previous page.)

Fig. 1 RHAMM ${ }^{B}$, but not RHAMM ${ }^{A}$, is upregulated in human PNETs and promotes liver metastasis of mouse PNET cells. a Diagram of RHAMM ${ }^{A}$ and $\mathrm{RHAMM}^{\mathrm{B}}$ proteins. $\mathbf{b}$ RHAMM is upregulated in 54 of 83 cases (65\%) of human PNETs in immunohistochemical staining. Left: Normal pancreas with islets in dashed circle. Middle: RHAMM negative PNET. Right: RHAMM positive PNET. Original magnification: $20 X$. Scale bar, $50 \mu m$. c RNA-seq analysis showed that RHAMM ${ }^{B}$ is significantly upregulated compared to RHAMM ${ }^{A}$ in primary human PNETs and liver metastases. The $p$ value was calculated using two-way ANOVA followed by Tukey's test. ${ }^{*}: p<0.0001, * * p<0.05$. Error bars represent standard error of mean. $\mathbf{d}$ Western blot analysis of human RHAMM in mouse N134 cell line (control), N134-RHAMM ${ }^{A}$ cells, and N134-RHAMM ${ }^{B}$ cells. e A total of 1 million N134 cells, N134-RHAMM ${ }^{A}$ cells, or N134-RHAMM ${ }^{B}$ cells were injected into the tail vein of NSG mice ( $n=5$ for each group). Five weeks later, the recipient mice were euthanized to survey for metastatic sites and incidence. Representative liver photos were shown

and $R H A M M^{B}$ by performing RNA-Seq analysis on 27 primary PNETs and 12 liver metastases, using 89 human islets from NCBI Gene Expression Omnibus database for comparison. Consistent with our immunohistochemical data, normal islets had very low $R H A M M^{A}$ and $R H A M M^{B}$ mRNA (Fig. 1c). RHAMM ${ }^{B}$ was significantly higher than $R H A M M^{A}$ in both primary and metastatic PNETs, suggesting that $R H A M M^{B}$ is the predominant isoform naturally expressed in PNETs. Although $R H A M M^{A}$ levels in primary tumors were significantly higher than those in normal islets $(p=0.0002), R H A M M^{A}$ levels in metastatic tumors were not significantly higher than those in normal islets $(p=0.8928)$. The mRNAs of RHAMM ${ }^{A}$ and $R H A M M^{B}$ were readily detectable in additional primary PNETs and metastases by RT-qPCR using isoform-specific primers (Additional file 1: Figure S1A-B).

We compared metastatic potential of $\mathrm{RHAMM}^{\mathrm{A}}$ to RHAMM $^{\mathrm{B}}$ in RIP-Tag; RIP-tva models of spontaneous metastasis and tail vein assays $[1,2]$. In contrast to $\operatorname{RHAMM}^{\mathrm{B}}$, RHAMM $^{\mathrm{A}}$ did not promote spontaneous metastasis (Additional file 2: Table S1). Then, we generated N134 cells overexpressing RHAMM $^{\mathrm{A}}\left(\mathrm{N} 134-\mathrm{RHAMM}^{\mathrm{A}}\right)$. N134 is a cell line derived from a PNET of RIP-Tag; RIP-tva mouse [1]. Although there were more RHAMM ${ }^{\mathrm{A}}$ than RHAMM ${ }^{\mathrm{B}}$ for unknown reasons (Fig. 1d), only one visible tumor was found in 5 immunodeficient NOD/scid-IL2Rgc knockout (NSG) mice receiving N134-RHAMM ${ }^{\mathrm{A}}$ cells after 5 weeks, while all 5 mice receiving N134-RHAMM ${ }^{B}$ cells developed large liver metastases within 5 weeks (Fig. 1e and Additional file 1: Figure S2A). To detect micrometastases, we performed immunostaining for synaptophysin, a neuroendocrine marker. Mice receiving N134 cells and N134-RHAMM ${ }^{\mathrm{A}}$ cells had an average of 1.8 and 0.6 liver micrometastases, respectively (Additional file 1 : Figure S2B). These data suggested that the unique 15-amino acid-stretch, ESQKNDKDLKILEKE, which is present in RHAMM $^{\mathrm{A}}$ but not in $\mathrm{RHAMM}^{\mathrm{B}}$, inhibits the metastatic function of RHAMM.

\section{RHAMM $^{B}$ is crucial for the metastatic potential of human PNET cell line BON1-TGL}

BON1 is the most utilized human PNET cell line and was established from a peri-pancreatic lymph node in a patient with metastatic PNET. We found that BON1 had much higher expression of $R H A M M^{B}$ than $R H A M M^{A}$ as determined by RNA-Seq (Fig. 2a). We performed shRNA-mediated knockdown of total RHAMM to investigate whether this reduces metastasis of BON1-TGL cells, which carry the thymidine kinase/green fluorescent protein /luciferase fusion reporter (TGL). Knockdown of RHAMM by shRNA was confirmed (Fig. 2b, c). We used an orthotopic model of PNET liver metastases by injecting cells into the spleen of NSG mice [6]. Mice receiving control cells developed an average of 82.5 liver metastases after 3 weeks, while mice receiving BON1-TGL-shRHAMM cells developed an average of 17 liver metastases with significantly lowered tumor burden (Fig. 2d-f).

To determine whether even higher RHAMM ${ }^{\mathrm{B}}$ levels would further enhance metastasis of BON1-TGL cells, we generated BON1-TGL cells overexpressing RHAMM ${ }^{\mathrm{B}}$ (Fig. 2g). We injected the cells into NSG mice via intracardiac injection. The increased levels of RHAMM $^{\mathrm{B}}$ enhanced metastasis of BON1-TGL in mice throughout the mouse body as visualized by bioluminescence imaging and signals from multiple organs were higher in mice receiving BON1-TGL-RHAMM ${ }^{\mathrm{B}}$ than those in mice receiving BON1-TGL overexpressing a control vector (Fig. 2h). Notably, we observed macrometastases at the adrenal glands of mice receiving BON1-TGL-RHAMM ${ }^{\mathrm{B}}$, but not in control mice (Fig. 2i). Taken together, RHAMM $^{B}$ is crucial for PNET metastasis.

\section{$R H A M M^{B}$ is upregulated in human pancreatic ductal} adenocarcinoma (PDAC) and correlates with poor survival PDAC is the most common pancreatic cancer type. It was shown that total $R H A M M$ is upregulated in primary PDAC by RT-qPCR of 14 matched tumors and adjacent normal tissues [7]. To compare RHAMM ${ }^{A}$ and $R H A M M^{B}$ levels in PDAC, we analyzed publicly available The Cancer Genome Atlas (TCGA) datasets. Both $R H A M M^{A}$ and $R H A M M^{B}$ were expressed at significantly higher levels in PDAC than in normal pancreatic tissues, and $R H A M M^{B}$ was substantially higher than $R H A M M^{A}$ (Fig. 3a). Survival analysis showed that high RHAMM levels were correlated with a worse outcome (Fig. 3b). Furthermore, patients with high $R H A M M^{B}$ had inferior survival compared to those with high $\operatorname{RHAMM}^{A}$ (Fig. 3c, d), suggesting that $R H A M M^{B}$, but not $R H A M M^{A}$, is a 

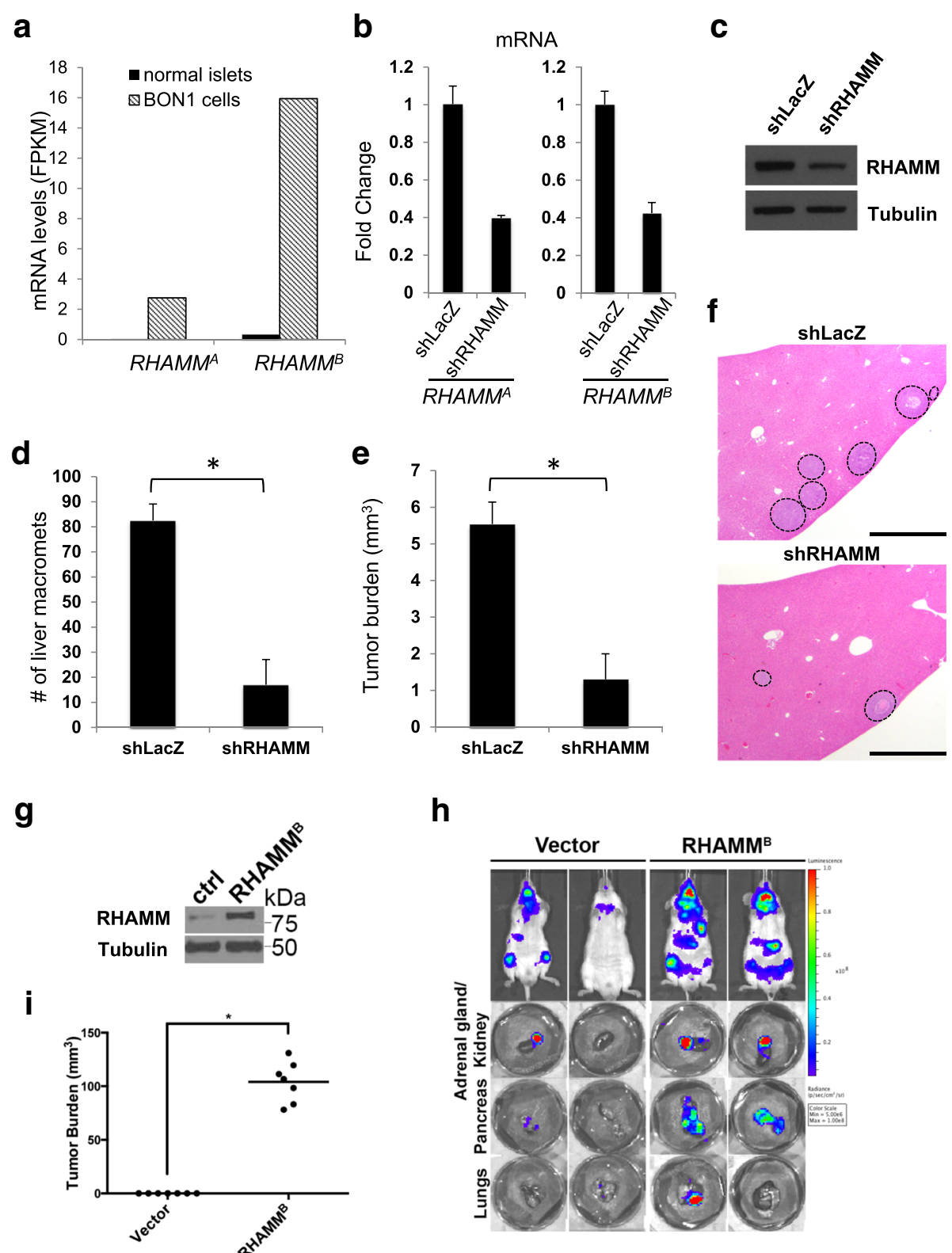

Fig. 2 RHAMM ${ }^{B}$ is crucial for metastatic potential of human PNET cell line, BON1-TGL. a RHAMM ${ }^{A}$ and $R H A M M^{B}$ expression in BON1 cell line compared to those in normal islets. b RHAMM ${ }^{A}$ and RHAMM ${ }^{B}$ knockdown in BON1-TGL cell line by shRHAMM as determined by RT-qPCR analysis. c Western blot analysis of RHAMM and tubulin (as a loading control) in BON1-TGL-shLacZ cell line and BON1-TGL-shRHAMM cell line. d-e RHAMM knockdown greatly inhibited liver metastasis of BON1-TGL cells. A total of 0.5 million each BON1-TGL-shLacZ (control) or BON1-TGL-shRHAMM were injected into the spleen of NSG mice ( $n=4$ for each group). After 3 weeks, the recipient mice were euthanized to survey for metastatic sites and incidence. The number of liver macrometastases (d) and the tumor burden of liver macrometastases (e) were recorded. *: statistically significantly different $(p<0.05$, one-tailed Mann-Whitney $\mathrm{U}$ test). Error bars represent standard deviation. $\mathbf{f}$ Liver sections with hematoxylin and eosin stain. Dashed circles indicate metastases. Original magnification: 10X. Scale bar, $1 \mathrm{~mm}$. g RHAMM ${ }^{B}$ overexpression in BON1-TGL-RHAMM ${ }^{B}$ cell line. Western blot analysis of RHAMM and tubulin (as a loading control) are shown. $\mathbf{h}$ Representative bioluminescent images of NSG mice 4 weeks after injection (upper panel) and their organs (lower panel). A total of 1 million cells was injected into NSG mice via intracardiac injection $(n=7)$. i The tumor burden of macrometastases at adrenal glands was documented. *: statistically significantly different, $p<0.05, \mathrm{t}$-test 
a

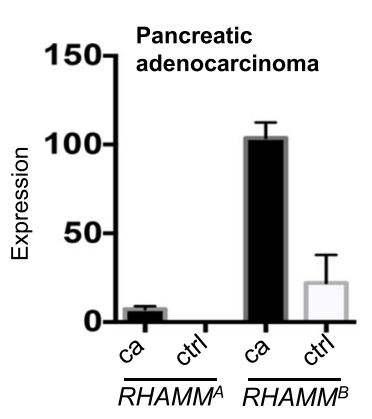

C

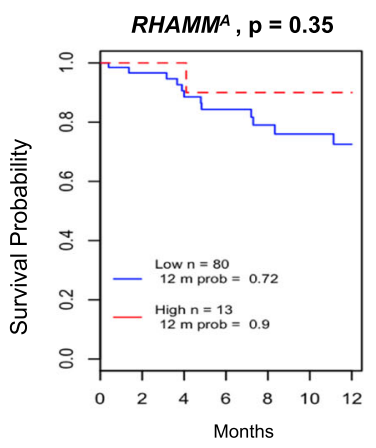

b Total RHAMM, $\mathrm{p}=\mathbf{0 . 0 3 4 5}$

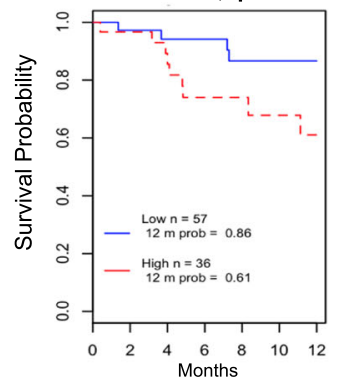

d

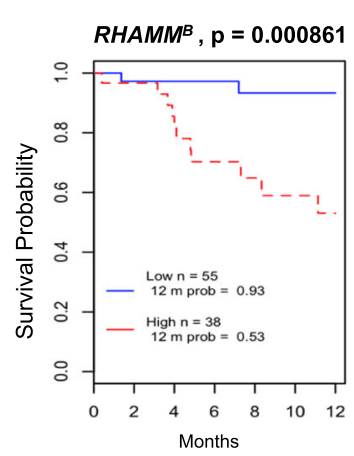

e

$f$
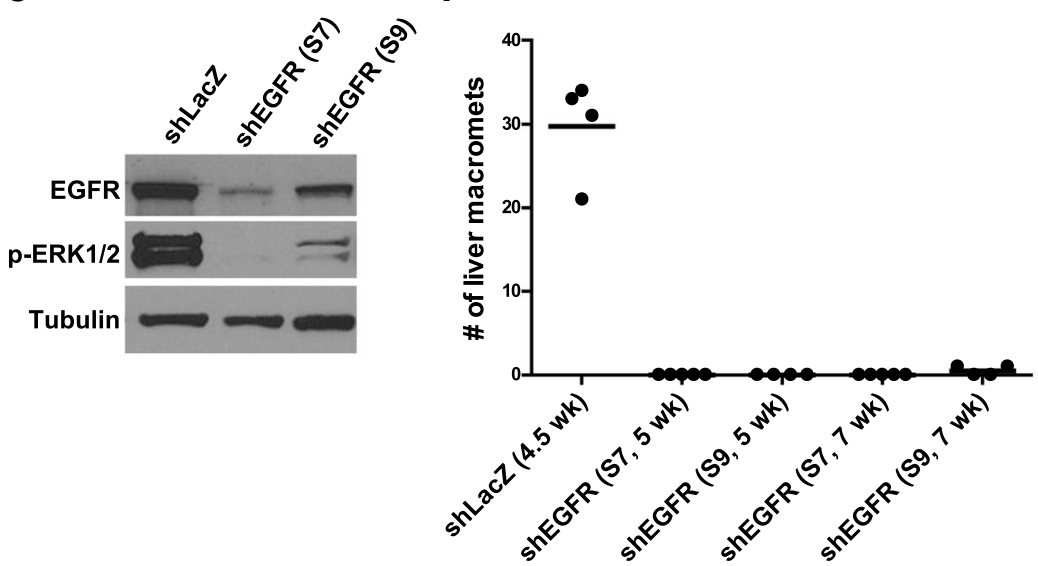

g

h

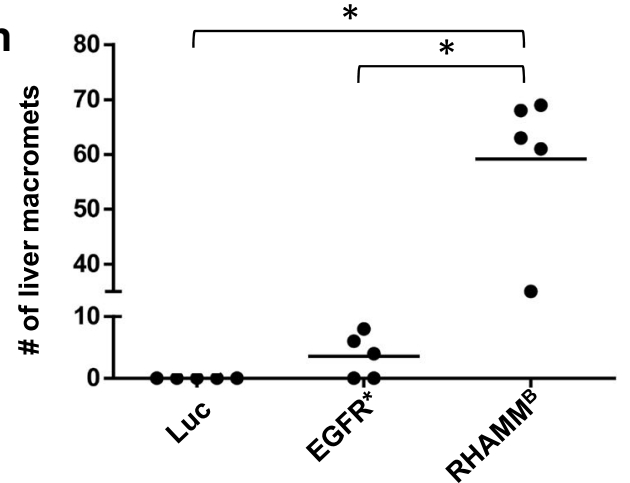

Fig. 3 (See legend on next page.) 
(See figure on previous page.)

Fig. 3 RHAMM ${ }^{B}$ is upregulated in human pancreatic ductal adenocarcinoma (PDAC) and correlates with poor survival. a RHAMM $^{A}$ and $R H A M M^{B}$ expression values from TCGA PDAC dataset. RHAMM ${ }^{A}$ : uc003lzf or NM_012484. RHAMM ${ }^{B}$ : uc003lzg or NM_012485. cancer (ca): $n=124 ;$ control (ctrl): $n=4$. Bars and error bars represent means and standard errors. b-d Kaplan-Meier survival analysis of TCGA cohort with 93 PDAC cases. High and low represent the status of the RHAMM mRNA expression levels compared to average values. e Knockdown efficiency of two EGFR shRNAs. S7 and S9 reduced EGFR protein expression and p-Erk1/2 levels in N134 cells overexpressing RHAMM ${ }^{B}$ by Western blot analysis. a-tubulin was used a loading control. $\mathbf{f}$ EGFR knockdown greatly inhibited the liver metastasis of N134 cells overexpressing RHAMM ${ }^{B}$. A total of 1 million

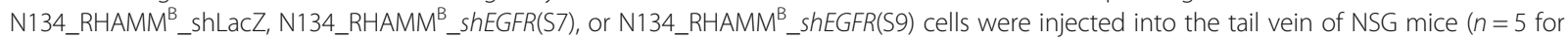
each group). At the indicated time points, the recipient mice were euthanized to survey for metastatic sites and incidence. The number of liver macrometastases was recorded. $\mathbf{g}$ Western blot analysis of p-Erk1/2 and total Erk from N134 overexpressing luciferase (control), and N134_EGFR*. h N134 cells overexpressing luciferase (Luc), N134_EGFR* (EGFR*), N134_RHAMM ${ }^{B}$ (RHAMM ${ }^{B}$ ) were injected into the tail vein of NSG mice $(n=5$, each group). Five weeks later (for Luc and EGFR* groups) or when mice were lethargic (for RHAMM ${ }^{\mathrm{B}}$ ), mice were euthanized to survey for metastatic sites and incidence. ${ }^{*}: p<0.0001$, One-way ANOVA and pairwise comparison with Tukey's adjustment

prognostic factor for survival of PDAC patients. Due to limited information available, we could not perform survival analysis for PNETs.

\section{EGFR signaling is required for $\mathrm{RHAMM}^{\mathrm{B}}$-induced metastasis}

EGFR activation is associated with worse survival in many malignancies. Analysis of TCGA PDAC dataset showed a good correlation between EGFR and RHAMM ${ }^{B}$ expression, but not between EGFR and $R H A M M^{A}$ expression (Additional file 1: Figure S3A-B). We previously showed that EGFR signaling is activated in N134-RHAMM ${ }^{B}$ cells and an EGFR inhibitor, gefitinib, induces apoptosis of N134-RHAMM ${ }^{B}$ cells [2]. These findings led us to hypothesize that enhanced EGFR signaling involves RHAMM ${ }^{\mathrm{B}}$-induced metastasis. To test this hypothesis, we used two different shRNAs targeting mouse EGFR (S7 and S9) as well as a control shRNA targeting LacZ (shLacZ). shEGFR(S7 and S9) decreased the levels of both EGFR and p-ERK1/2, a downstream target of EGFR signaling, with $\mathrm{S7}$ exhibiting a better knockdown efficiency than S9 (Fig. 3e).

In tail vein metastasis assays, mice receiving N134-RHAMM ${ }^{\mathrm{B}}$-shLacZ became lethargic, showing a mean of 29.8 liver macrometastases per mouse within 5 weeks, but mice injected with N134-RHAMM ${ }^{\mathrm{B}}-\operatorname{shEGFR(S7)}$ and N134-RHAMM ${ }^{\mathrm{B}}$-shEGFR(S9) cells did not develop metastases 5 weeks post-injection (Fig. 3f). An additional cohort of mice injected with N134-RHAMM ${ }^{\mathrm{B}}$-shEGFR(S7) still did not develop metastases 7 weeks post-injection, and only a mean of 0.5 liver macrometastases appeared in mice injected with N134-RHAMM ${ }^{\mathrm{B}}$-shEGFR(S9) at this time point (Fig. 3f).

We investigated whether a constitutively active form of EGFR, EGFR*, is sufficient to recapitulate RHAMM ${ }^{B}$ activity in metastasis. Five of the 8 RIP-Tag; RIP-tva mice receiving RCASBP-EGFR* developed pancreatic lymph node metastases (62.5\%) and 2 developed liver metastases (25\%) (Additional file 2: Table S1). We generated N134 cells overexpressing EGFR* (N134-EGFR*) for experimental metastasis (Fig. 3g). While no metastasis was found in mice receiving control N134-Luciferase after 6 weeks, an average of 6 liver macrometastases was detected in mice receiving N134-EGFR* (Fig. 3h). Although EGFR* promotes liver metastasis of PNETs in both spontaneous and experimental metastasis mouse models, the degree of metastasis is less than that of RHAMM ${ }^{\mathrm{B}}$ (Additional file 2: Table S1 and Fig. 3h). Therefore, these EGFR knockdown and EGFR* overexpression data suggest that activation of EGFR contributes to RHAMM ${ }^{\mathrm{B}}$-induced PNET metastasis, but EGFR* cannot fully recapitulate the metastatic phenotype of RHAMM $^{\mathrm{B}}$. Further studies are required to identify signals other than EGFR provided by RHAMM $^{\mathrm{B}}$ and to understand whether endogenous levels of RHAMM $^{\mathrm{A}}$ activate EGFR signaling.

\section{Conclusion}

We provide evidence that upregulation of RHAMM ${ }^{\mathrm{B}}$ is a valuable prognostic marker for pancreatic cancer. We demonstrated that only the shorter isoform RHAMM $^{\mathrm{B}}$, but not RHAMM ${ }^{\mathrm{A}}$ with 15 extra amino acids encoded by exon 4, is significantly upregulated in PNET and PDAC. RHAMM $^{\mathrm{B}}$, but not RHAMM ${ }^{\mathrm{A}}$, promotes metastasis in spontaneous and experimental metastasis mouse models of PNET. EGFR signaling is required for RHAMM ${ }^{\mathrm{B}}$-induced liver metastasis, but is not sufficient to promote PNET metastasis. $R H A M M^{B}$, but not $R H A M M^{A}$, is correlated with inferior survival in PDAC patients.

\section{Additional files}

\begin{abstract}
Additional file 1: Figure S1. RT-qPCR analysis of $\operatorname{RHAMM}^{A}(\mathrm{~A})$ and RHAMM $^{B}(B)$ in 9 primary human PNETs and 3 metastatic PNETs from the livers. Figure S2. Unlike RHAMM ${ }^{B}, \mathrm{RHAMM}^{\mathrm{A}}$ did not promote liver metastasis of mouse PNET N134 cell line in a tail vein experimental metastasis assay. (A) The number of liver macrometastases was recorded. (B) Immunohistochemical staining of synaptophysin in the liver sections to reveal the presence of metastatic PNETs. Arrows indicate micrometastases. Original magnification: 10X. Scale bar, $200 \mu \mathrm{m}$. Figure S3. Correlation between EGFR expression and RHAMM $^{A}(\mathrm{~A})$ or RHAMM ${ }^{B}$ (B) expression in TCGA PDAC dataset (RNA-Seq V2). (PPTX $3371 \mathrm{~kb}$ )
\end{abstract}

Additional file 2: Table S1. Impact of genes on lymph node and liver metastasis of PNETs in RIP-Tag; RIP-tva mice. (DOCX 37 kb)

Additional file 3: Supplementary materials and methods. (DOCX $37 \mathrm{~kb}$ ) 


\section{Abbreviations}

DMEM: Dulbecco's modified Eagle's medium; FBS: Fetal bovine serum; FPKM: Fragments per kilobase of transcripts per million reads; HA: Hyaluronic acid; PDAC: Pancreatic ductal adenocarcinoma; PNET: Pancreatic neuroendocrine tumor; RHAMM: Receptor for hyaluronic acid-mediated motility; RT-qPCR: Quantitative real-time reverse transcription PCR; TCGA: The Cancer Genome Atlas (TCGA)

\section{Acknowledgments}

The authors thank Danny Huang for mouse database design; Harold Varmus, Yi Li, Jihye Paik, Todd R. Evans, David Foster, Bi-Sen Ding, Katie Politi, Jeffrey Yongchun Zhao, Romel Somwar, Selina Chen-Kiang, Stephanie Azzopardi, Samantha Li, Megan Wong, Joseph Na, and Robin Zhang for their valuable input and excellent assistance; Diane L. Reidy and David S. Klimstra for contributing the approval of the tumor materials.

\section{Funding}

This work is partially supported by NIH grants 2U01DK072473 (to Y.-C.N.D.), 1R21CA173348-01A1 (to Y.-C.N.D.), 1 R01CA204916-01A1 (to Z.C., G.Z., Y.C.N.D.), UL1TR000457 (to Z.C.), DOD grants W81XWH-13-1-0331 (to S.C., Z.C., Y.-C.N.D.) W81XWH-16-1-0619 (to Y.-C.N.D.), and Goldhirsh Foundation (to A.V., T.J. F.III, O.E., Y.-C.N.D.).

\section{Availability of data and materials}

The RNA-Seq and TCGA datasets that support the findings of this study are available in Gene Expression Omnibus (GEO accession GSE50398, http:// www.ncbi.nlm.nih.gov/geo/query/acc.cgi?acc=GSE50398) [8-10], and http:// cancergenome.nih.gov/cancersselected, and the other data are available from the authors upon reasonable request.

\section{Authors' contributions}

SC, XC, BJK, GZ, SP, TH, and LS performed the experiments and analyzed the data. DW conducted the analysis on the TCGA dataset. DW, GZ, SP, TJFIII, and KR edited the manuscript. LHT contributed immunohistochemical interpretation. AV, CSC, and OE contributed to bioinformatics analyses. ZC performed statistical analysis. LHT, KR, and TJFIII contributed to samples. $\mathrm{Y}$-CND designed and performed the experiments, analyzed the data, and wrote the manuscript. All authors read and approved the final manuscript.

\section{Ethics approval and consent to participate}

All procedures involving mice were approved by the institutional animal care and use committee. Retrospective and prospective review of PNETs was performed using the pathology files and pancreatic cancer database at the authors' institutions with IRB approval. Experimental details are provided in Additional file 3: Supplementary materials and methods.

\section{Consent for publication}

All authors agreed on the manuscript.

\section{Competing interests}

The authors declare that they have no competing interests.

\section{Publisher's Note}

Springer Nature remains neutral with regard to jurisdictional claims in published maps and institutional affiliations.

\section{Author details}

'Department of Pathology and Laboratory Medicine, Weill Cornell Medicine, Box 69, New York, NY 10065, USA. ${ }^{2}$ Laboratory of Cellular Oncology, National Cancer Institute, National Institutes of Health, Bethesda, MD 20892, USA. ${ }^{3}$ Department of Pathology, Memorial Sloan Kettering Cancer Center, New York, NY 10065, USA. ${ }^{4}$ Caryl and Israel Englander Institute for Precision Medicine, Institute for Computational Biomedicine, Department of Physiology and Biophysics, Weill Cornell Medicine, New York, NY 10065, USA. ${ }^{5}$ Division of Biostatistics and Epidemiology, Department of Healthcare Policy and Research, Weill Cornell Medicine, New York, NY 10065, USA. 'Weill Cornell Graduate School of Medical Sciences, Cornell University, New York, NY 10065, USA. ${ }^{7}$ Rutgers Cancer Institute of New Jersey, New Brunswick, NJ 08903, USA. ${ }^{8}$ Department of Surgery, Weill Cornell Medicine, New York, NY 10065, USA.
Received: 28 December 2018 Accepted: 10 April 2019

Published online: 09 May 2019

\section{References}

1. Du YC, Lewis BC, Hanahan D, Varmus H. Assessing tumor progression factors by somatic gene transfer into a mouse model: BCl-xL promotes islet tumor cell invasion. PLoS Biol. 2007;5:e276.

2. Du YC, Chou CK, Klimstra DS, Varmus H. Receptor for hyaluronan-mediated motility isoform B promotes liver metastasis in a mouse model of multistep tumorigenesis and a tail vein assay for metastasis. Proc Natl Acad Sci U S A. 2011;108:16753-8.

3. Greiner J, Ringhoffer M, Taniguchi M, Schmitt A, Kirchner D, Krahn G, Heilmann V, Gschwend J, Bergmann L, Dohner H, Schmitt M. Receptor for hyaluronan acid-mediated motility (RHAMM) is a new immunogenic leukemia-associated antigen in acute and chronic myeloid leukemia. Exp Hematol. 2002;30:1029-35.

4. Chen YT, Chen Z, Du YN. Immunohistochemical analysis of RHAMM expression in normal and neoplastic human tissues: a cell cycle protein with distinctive expression in mitotic cells and testicular germ cells. Oncotarget. 2018;9:20941-52.

5. Sironen RK, Tammi M, Tammi R, Auvinen PK, Anttila M, Kosma VM. Hyaluronan in human malignancies. Exp Cell Res. 2011;317:383-91.

6. Fraedrich K, Schrader J, Ittrich H, Keller G, Gontarewicz A, Matzat V, Kromminga A, Pace A, Moll J, Blaker M, et al. Targeting aurora kinases with danusertib (PHA-739358) inhibits growth of liver metastases from gastroenteropancreatic neuroendocrine tumors in an orthotopic xenograft model. Clin Cancer Res. 2012;18:4621-32.

7. Cheng XB, Sato N, Kohi S, Koga A, Hirata K. Receptor for hyaluronic acidmediated motility is associated with poor survival in pancreatic ductal adenocarcinoma. J Cancer. 2015;6:1093-8.

8. Zhou Y, Park SY, Su J, Bailey K, Ottosson-Laakso E, Shcherbina L, Oskolkov N, Zhang $E$, Thevenin T, Fadista J, et al. TCF7L2 is a master regulator of insulin production and processing. Hum Mol Genet. 2014;23:6419-31.

9. Fadista J, Vikman P, Laakso EO, Mollet IG, Esguerra JL, Taneera J, Storm P, Osmark P, Ladenvall C, Prasad RB, et al. Global genomic and transcriptomic analysis of human pancreatic islets reveals novel genes influencing glucose metabolism. Proc Natl Acad Sci U S A. 2014;111:13924-9.

10. Taneera J, Fadista J, Ahlqvist E, Atac D, Ottosson-Laakso E, Wollheim CB, Groop L. Identification of novel genes for glucose metabolism based upon expression pattern in human islets and effect on insulin secretion and glycemia. Hum Mol Genet. 2015;24:1945-55.

\section{Ready to submit your research? Choose BMC and benefit from:}

- fast, convenient online submission

- thorough peer review by experienced researchers in your field

- rapid publication on acceptance

- support for research data, including large and complex data types

- gold Open Access which fosters wider collaboration and increased citations

- maximum visibility for your research: over $100 \mathrm{M}$ website views per year

At BMC, research is always in progress.

Learn more biomedcentral.com/submissions 